\title{
The Frobenius Complex
}

\author{
Eric Clark and Richard Ehrenborg
}

Department of Mathematics, University of Kentucky, Lexington, KY 40506-0027

\begin{abstract}
Motivated by the classical Frobenius problem, we introduce the Frobenius poset on the integers $\mathbb{Z}$, that is, for a sub-semigroup $\Lambda$ of the non-negative integers $(\mathbb{N},+)$, we define the order by $n \leq_{\Lambda} m$ if $m-n \in \Lambda$. When $\Lambda$ is generated by two relatively prime integers $a$ and $b$, we show that the order complex of an interval in the Frobenius poset is either contractible or homotopy equivalent to a sphere. We also show that when $\Lambda$ is generated by the integers $\{a, a+d, a+2 d, \ldots, a+(a-1) d\}$, the order complex is homotopy equivalent to a wedge of spheres.

Résumé. Motivé par le problème de Frobenius classique, nous introduisons l'ensemble partiellement ordonné de Frobenius sur les entiers $\mathbb{Z}$, c.à.d. que pour un sous-semigroupe $\Lambda$ de les entiers non-négatifs $(\mathbb{N},+)$ nous définissons l'ordre par $n \leq_{\Lambda} m$ si $m-n \in \Lambda$. Quand le $\Lambda$ est engendré par deux nombres $a$ et $b$, relativement premiers entre eux, noux montrons que le complexe des chaînes d'un intervalle quelquonque dans l'ensemble partiellement ordonné de Frobenius est soit contractible soit homotopiquement équivalent à une sphère. Nous montrons aussi que dans le cas où $\Lambda$ est engendré par les entiers $\{a, a+d, a+2 d, \ldots, a+(a-1) d\}$, le complexe des chaînes a le type de homotopie d'un bouquet de sphères.
\end{abstract}

Keywords: order complex, homotopy type, Morse matching, cylindrical posets

\section{Introduction}

The classical Frobenius problem is to find the largest integer for which change cannot be made using coins with the relatively prime denominations $a_{1}, a_{2}, \ldots, a_{d}$; see for instance [2, Section 1.2]. We will reformulate this question by introducing the following poset.

Let $\Lambda$ be a sub-semigroup of the non-negative integers $\mathbb{N}$, that is, $\Lambda$ is closed under addition and the element 0 lies in $\Lambda$. We define the Frobenius poset $P=\left(\mathbb{Z}, \leq_{\Lambda}\right)$ on the integers $\mathbb{Z}$ by the order relation $n \leq_{\Lambda} m$ if $m-n \in \Lambda$. We denote by $[n, m]_{\Lambda}$ the interval from $n$ to $m$ in the Frobenius poset, that is,

$$
[n, m]_{\Lambda}=\{i \in[n, m]: i-n, m-i \in \Lambda\}
$$

Observe that the interval $[n, m]_{\Lambda}$ in the Frobenius poset is isomorphic to the interval $[n+i, m+i]_{\Lambda}$, that is, the interval $[n, m]_{\Lambda}$ only depends on the difference $m-n$. Also note that each interval is self-dual by sending $i$ in $[0, n]_{\Lambda}$ to $n-i$.

In this form, the original Frobenius problem would be to find the largest integer $n$ that is not comparable to zero in the Frobenius poset when $\Lambda$ is generated by $\left\{a_{1}, a_{2}, \ldots, a_{d}\right\}$. The largest such integer is known as the Frobenius number. In general, calculating the Frobenius number is difficult. However, in the case where the semigroup is generated by two relatively prime integers $a$ and $b$, it is well known that 


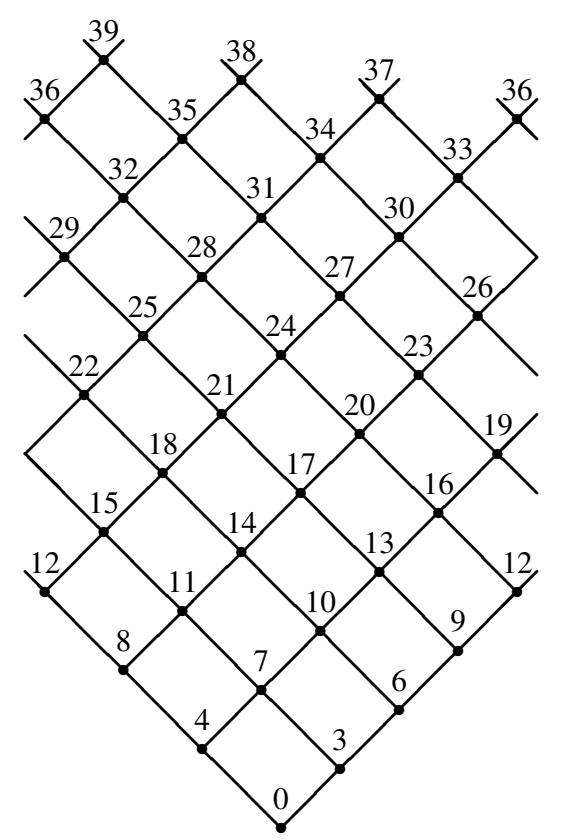

Fig. 1: The filter generated by 0 in the Frobenius poset corresponding to the semigroup $\Lambda$ generated by $a=3$ and $b=4$, that is, $\Lambda=\mathbb{N}-\{1,2,5\}$. Note that you get a better picture by rolling the page into a cylinder.

the Frobenius number is given by $a b-a-b$. Also, when the semigroup is generated by the arithmetic sequence $\{a, a+d, \ldots, a+s d\}$, the Frobenius number was shown by Roberts [17] to be

$$
\left(\left\lfloor\frac{a-2}{s}\right\rfloor+1\right) \cdot a+(d-1)(a-1)-1
$$

We study the topology of the order complex of intervals of this poset in the two generator case and when the generators form an arithmetic sequence where $s=a-1$.

The technique we use is discrete Morse theory which was developed by Forman [8, 9]. Thus we construct an acyclic partial matching on the face poset of the order complex by using the Patchwork Theorem. We then identify the unmatched, or critical, cells. These tell us the number and dimension of cells in a CW-complex to which our order complex is homotopy equivalent. Using extra structure about the critical cells, we can determine exactly what the homotopy type is.

A more general situation is to consider a semigroup $\Lambda$ of $\mathbb{N}^{d}$ and define a partial order on $\mathbb{Z}^{d}$ by $\mu \leq_{\Lambda} \lambda$ if $\lambda-\mu \in \Lambda$. Define the semigroup algebra $k[\Lambda]$ as the linear span of the monomials whose powers belong to $\Lambda$, that is, $k[\Lambda]=\operatorname{span}\left\{x^{\lambda}=x_{1}^{\lambda_{1}} \cdots x_{d}^{\lambda_{d}}: \lambda \in \Lambda\right\}$. Laudal and Sletsjøe [14] makes the connection between the homology of the order complex of intervals in this partial order and the semigroup algebra $k[\Lambda]$.

Theorem 1.1 (Laudal and Sletsjøe) For $\Lambda$ a sub-semigroup of $\mathbb{N}^{d}$ with the associated monoid $\Lambda$, the following equality holds

$$
\operatorname{dim}_{k} \operatorname{Tor}_{i}^{k[\Lambda]}(k, k)_{\lambda}=\operatorname{dim}_{k} \widetilde{H}_{i-2}\left(\Delta\left([0, \lambda]_{\Lambda}\right), k\right),
$$


for all $\lambda \in \Lambda$ and $i \geq 0$.

The papers [5, 10, 16] continue to study the topology of the intervals in this partial order. Hersh and Welker [10] give bounds on the indices of the non-vanishing homology groups of the order complex of the intervals. Peeva, Reiner, and Sturmfels [16] show that the semigroup ring $k[\Lambda]$ is Koszul if and only if each interval in $\Lambda$ is Cohen-Macaulay.

We end this paper with some open questions and concluding remarks.

\section{Discrete Morse theory}

We recall the following definitions and theorems from discrete Morse theory. See [8, 9, 12] for further details.

Definition 2.1 A partial matching in a poset $P$ is a partial matching in the underlying graph of the Hasse diagram of $P$, that is, a subset $M \subseteq P \times P$ such that $(x, y) \in M$ implies $x \prec y$ and each $x \in P$ belongs to at most one element of $M$. For $(x, y) \in M$ we write $x=d(y)$ and $y=u(x)$, where $d$ and $u$ stand for down and up, respectively.

Definition 2.2 A partial matching $M$ on $P$ is acyclic if there does not exist a cycle

$$
z_{1} \succ d\left(z_{1}\right) \prec z_{2} \succ d\left(z_{2}\right) \prec \cdots \prec z_{n} \succ d\left(z_{n}\right) \prec z_{1},
$$

in $P$ with $n \geq 2$, and all $z_{i} \in P$ distinct. Given a partial matching, the unmatched elements are called critical. If there are no critical elements, the acyclic matching is perfect.

We now state the main result from discrete Morse theory. For a simplicial complex $\Delta$, let $\mathcal{F}(\Delta)$ denote the poset of faces of $\Delta$ ordered by inclusion.

Theorem 2.3 Let $\Delta$ be a simplicial complex. If $M$ is an acyclic matching on $\mathcal{F}(\Delta)-\{\widehat{0}\}$ and $c_{i}$ denotes the number of critical $i$-dimensional cells of $\Delta$, then the complex $\Delta$ is homotopy equivalent to a $C W$ complex $\Delta_{c}$ which has $c_{i}$ cells of dimension $i$.

For us it will be convenient to work with the reduced discrete Morse theory, that is, we include the empty set.

Corollary 2.4 Let $\Delta$ be a simplicial complex and let $M$ be an acyclic matching on $\mathcal{F}(\Delta)$. Then the space $\Delta$ is homotopy equivalent to a CW complex $\Delta_{c}$ which has $c_{0}+1$ cells of dimension 0 and $c_{i}$ cells of dimension i for $i>0$.

In particular, if the matching from Corollary 2.4 is perfect, then $\Delta_{c}$ is contractible. Also, if the matching has exactly one critical cell then $\Delta_{c}$ is a combinatorial $d$-sphere where $d$ is the dimension of the cell.

Given a set of critical cells of differing dimension, in general it is impossible to conclude that the CW complex $\Delta_{c}$ is homotopy equivalent to a wedge of spheres. See Kozlov [13] for an example. However, in certain cases, this is possible.

Theorem 2.5 Let $M$ be a Morse matching on $\mathcal{F}(\Delta)$ such that all $c_{i}$ critical cells of dimension $i$ are maximal. Then

$$
\Delta \simeq \bigvee_{i} \bigvee_{j=1}^{c_{i}} \mathbb{S}^{i}
$$


Proof: By the above statement, the complex $\Delta$ without the critical cells is contractible. In particular, the boundary of each of the critical cells contracts to a point. Since all of the critical cells are maximal, they can be independently added back into the complex.

Kozlov [13] gives a more general sufficient condition on an acyclic Morse matching for the complex to be homotopy equivalent to a wedge of spheres enumerated by the critical cells.

We are interested in finding an acyclic matching on the face poset of the Frobenius complex. The Patchwork Theorem [12] gives us a way of constructing one.

Theorem 2.6 Assume that $\varphi: P \rightarrow Q$ is an order-preserving poset map, and assume that there are acyclic matchings on the fibers $\varphi^{-1}(q)$ for all $q \in Q$. Then the union of these matchings is itself an acyclic matching on $P$.

\section{Two generators}

With two generators, the associated Frobenius poset can be embedded on a cylinder. By Bezout's identity there are two integers $p$ and $q$ such that $p \cdot a+q \cdot b=1$. Define a group morphism $\gamma: \mathbb{Z} \longrightarrow \mathbb{Z}_{2 a b} \times \mathbb{Z}$ by $\gamma(x)=((p \cdot a-q \cdot b) \cdot x, x)$, that is, the first coordinate is modulo $2 \cdot a \cdot b$ which corresponds to encircling the cylinder. Observe that $\gamma(a)=((p \cdot a-q \cdot b) \cdot a, a)=((p \cdot a+q \cdot b) \cdot a, a)=(a, a)$ and $\gamma(b)=((p \cdot a-q \cdot b) \cdot b, b)=((-p \cdot a-q \cdot b) \cdot b, b)=(-b, b)$. Hence the two cover relations $x \prec x+a$ and $x \prec x+b$ in the Frobenius poset translates to $\gamma(x)+(a, a)=\gamma(x+a)$ and $\gamma(x)+(-b, b)=\gamma(x+b)$. In other words, to take an $a$ step we make the step $(a, a)$ on the cylinder and a $b$ step corresponds to the step $(-b, b)$. As an example, see Figure 1 where $a=3$ and $b=4$.

In general, the Frobenius poset is not a lattice. When $\Lambda$ is generated by two relatively prime integers $a$ and $b$, we have the four relations $a<_{\Lambda} a+b, b<_{\Lambda} a+b, a<_{\Lambda} a b$, and $b<_{\Lambda} a b$. However, since $a b-a-b$ is the Frobenius number we have $a+b \not_{\Lambda} a b$, showing that the poset is not a lattice. In Figure 1, we see that 3 and 4 are both lower bounds for 7 and 12 .

Let $c_{k}(n)$ denote the number of chains in the Frobenius interval $[0, n]_{\Lambda}$ of length $k$. Using multiplication of generating functions, we have

$$
\sum_{n \geq k} c_{k}(n) \cdot q^{n}=\left(\sum_{n \geq 1} c_{1}(n) \cdot q^{n}\right)^{k} .
$$

By taking the alternating sum over $k$ and using Philip Hall's expression for the Möbius function, we have

$$
\sum_{n \geq 0} \mu(n) \cdot q^{n}=\frac{1}{1+\sum_{n \geq 1} c_{1}(n) \cdot q^{n}}
$$

where $\mu(n)$ denotes the Möbius function of the interval $[0, n]_{\Lambda}$. Now assuming that $\Lambda$ is generated by two relatively prime positive integers $a$ and $b$, we have that

$$
1+\sum_{n \geq 1} c_{1}(n) \cdot q^{n}=\frac{1-q^{a b}}{\left(1-q^{a}\right) \cdot\left(1-q^{b}\right)} ;
$$


see [1, Exercise VIII.1.5]. Hence the Möbius function is given by

$$
\begin{aligned}
\sum_{n \geq 0} \mu(n) \cdot q^{n} & =\frac{\left(1-q^{a}\right) \cdot\left(1-q^{b}\right)}{1-q^{a b}} \\
& =1-q^{a}-q^{b}+q^{a+b}+q^{a b}-q^{a b+a}-q^{a b+b}+q^{a b+a+b}+\cdots .
\end{aligned}
$$

Note that the coefficients are all \pm 1 or 0 . We will consider this fact in a topological setting. Recall that the order complex $\Delta(P)$ of a bounded poset $P$ is the collection of chains in $P$, that is,

$$
\Delta(P)=\left\{\left\{x_{1}, x_{2}, \ldots, x_{k}\right\}: \widehat{0}<x_{1}<x_{2}<\cdots<x_{k}<\widehat{1}\right\}
$$

ordered by inclusion. Also, the reduced Euler characteristic of the order complex $\Delta(P)$ is given by the Möbius function of $P$. We call the order complex of the face poset of a Frobenius interval the Frobenius complex. We wish to study the homotopy type of the Frobenius complex. Since the reduced Euler characteristic of the Frobenius complex takes on the values $+1,-1$, or 0 , we are lead to the following main theorem.

Theorem 3.1 Let the sub-semigroup $\Lambda$ be generated by two relatively prime positive integers a and $b$ with $1<a<b$. The order complex of the associated Frobenius interval $[0, n]_{\Lambda}$, for $n \geq 1$, is homotopy equivalent to either a sphere or contractible, according to

$$
\Delta\left([0, n]_{\Lambda}\right) \simeq\left\{\begin{array}{cl}
\mathbb{S}^{2 n / a b-2} & \text { if } n \equiv 0 \bmod a \cdot b, \\
\mathbb{S}^{2(n-a) / a b-1} & \text { if } n \equiv a \bmod a \cdot b, \\
\mathbb{S}^{2(n-b) / a b-1} & \text { if } n \equiv b \bmod a \cdot b, \\
\mathbb{S}^{2(n-a-b) / a b} & \text { if } n \equiv a+b \bmod a \cdot b, \\
\text { point } & \text { otherwise. }
\end{array}\right.
$$

Observe that if $n$ does not belong to the sub-semigroup $\Lambda$ then we consider the order complex $\Delta\left([0, n]_{\Lambda}\right)$ to be the empty set which we view as contractible. This is distinct from the case when $n$ equals $a$ or $b$, that is, when the order complex $\Delta\left([0, n]_{\Lambda}\right)$ only contains the empty set. In this case, we view this as a sphere of dimension -1 .

In the case where the two generators are 2 and 3 , the semigroup is $\mathbb{N}-\{1\}$ and the order complex $\Delta\left([0, n]_{\Lambda}\right)$ consists of all subsets of the interval $[2, n-2]$ that do not contain two consecutive integers. This is known as the complex of sparse subsets. Its homotopy type was first determined by Kozlov [11]. See also [7] where it appears as the independence complex of a path. Billera and Myers [4] showed this complex is non-pure shellable.

As a corollary to Theorem 1.1 , we obtain

Corollary 3.2 Let $a$ and $b$ be relatively prime integers such that $1<a<b$. Let $R$ denote the ring $k[y, z] /\left(y^{b}-z^{a}\right)$. Then the multigraded Poincaré series

$$
P_{k}^{R}(t, q)=\sum_{n \in \Lambda} \sum_{i \geq 0} \operatorname{dim}_{k}\left(\operatorname{Tor}_{i}^{R}(k, k)_{n}\right) t^{i} q^{n}
$$

is given by the rational function

$$
\frac{1+t q^{a}+t q^{b}+t^{2} q^{a+b}}{1-t^{2} q^{a b}}
$$


Proof: Let $\Lambda$ the semigroup generated by $a$ and $b$. Observe that the ring $R$ is isomorphic to the semigroup ring $k[\Lambda]$. By combining Theorems 1.1 and 3.1 the multigraded Poincaré series is given by

$$
P_{k}^{R}(t, q)=1+t q^{a}+t q^{b}+t^{2} q^{a+b}+t^{2} q^{a b}+t^{3} q^{a b+a}+t^{3} q^{a b+b}+t^{4} q^{a b+a+b}+\cdots,
$$

which is the sought after rational generating function.

We now turn our attention to the proof of Theorem 3.1. Let $\Lambda$ be generated by two relatively prime positive integers $a$ and $b$ with $1<a<b$. Consider the Frobenius interval $[0, n]_{\Lambda}$. Define the three sets $B_{\ell}, C_{\ell}$ and $D_{\ell}$ as follows:

$$
\begin{aligned}
& B_{\ell}=\{\ell a b+2 b, \ell a b+3 b, \ldots, \ell a b+(a-1) b\}, \\
& C_{\ell}=\{b, a b, a b+b, 2 a b, 2 a b+b, 3 a b, \ldots,(\ell-1) a b+b, \ell a b\}, \\
& D_{\ell}=C_{\ell} \cup\{\ell a b+b\} .
\end{aligned}
$$

Note that $C_{0}=\emptyset, D_{0}=\{b\}$, and $C_{\ell+1}=D_{\ell} \cup\{(\ell+1) a b\}$.

Let $Q$ be the infinite chain $\{a<a+b<a b+a<a b+a+b<2 a b+a<\cdots\}$ adjoined with a new maximal element $\widehat{1}_{Q}$, that is,

$$
Q=\{m \in \mathbb{N}: m \equiv a, a+b \bmod a b\} \cup\left\{\widehat{1}_{Q}\right\} .
$$

We now define a map $\varphi$ from the face poset of the order complex $\Delta\left([0, n]_{\Lambda}\right)$ to the poset $Q$. We will later show that $\varphi$ is an order-preserving poset map with natural matchings on the fibers. Let $\varphi$ be defined by

$$
\varphi(x)=\left\{\begin{array}{cl}
\ell a b+a & \text { if } \ell a b+a<_{\Lambda} n, \\
& C_{\ell} \subseteq x, \\
& B_{t} \cap x=\emptyset \text { for } 0 \leq t \leq \ell, \\
& \text { and } \ell a b+b \notin x ; \\
\ell a b+a+b & \text { if } \ell a b+a+b<_{\Lambda} n, \\
& D_{\ell} \subseteq x, \\
& B_{t} \cap x=\emptyset \text { for } 0 \leq t \leq \ell, \\
& \text { and } \ell a b+a b \notin x ; \\
\widehat{1}_{Q} & \text { otherwise. }
\end{array}\right.
$$

In order to make acyclic pairings on the fibers of $\varphi$, it will be useful to have a description of the chains that are mapped to the maximal element $\widehat{1}_{Q}$ and their structure. Let $\Gamma$ denote this collection of chains in the Frobenius poset, that is, $\Gamma=\varphi^{-1}\left(\widehat{1}_{Q}\right)$.

Lemma 3.3 The collection $\Gamma$ consists of the chains $x$ that satisfy one of the following four conditions:

1. There exists a non-negative integer $\lambda$ such that $C_{\lambda} \subseteq x, \lambda a b+b \notin x, B_{\lambda} \cap x \neq \emptyset$, and $B_{t} \cap x=\emptyset$ for $0 \leq t \leq \lambda-1$.

2. There exists a non-negative integer $\lambda$ such that $D_{\lambda} \subseteq x, B_{\lambda} \cap x \neq \emptyset$, and $B_{t} \cap x=\emptyset$ for $0 \leq t \leq \lambda-1$.

3. There exists a non-negative integer $\lambda$ such that $x=C_{\lambda}$ and $\lambda a b+a \nless_{\Lambda} n$. 
4. There exists a non-negative integer $\lambda$ such that $x=D_{\lambda}$ and $\lambda a b+a+b \nless_{\Lambda} n$.

We will refer to the condition met by a chain as its type and the associated $\lambda$ as its parameter. The structure of $\Gamma$ is given in the following lemma.

Lemma 3.4 The following four conditions hold for the collection $\Gamma$.

(i) Let $x$ be a chain of type 1 with parameter $\lambda$ in $\Gamma$. Then $x \cup\{\lambda a b+b\}$ is a chain in $\Gamma$ of type 2 with the same parameter $\lambda$.

(ii) Let $y$ be a chain of type 2 with parameter $\lambda$ in $\Gamma$. Then $y-\{\lambda a b+b\}$ is a chain in $\Gamma$ of type 1 with the same parameter $\lambda$.

(iii) Let $x$ be a chain of type 1 with parameter $\lambda$ and $y$ be a chain of type 2 with parameter $\mu$ such that $x \prec y$. Then $\lambda \geq \mu$ holds with equality if and only if $y=x \cup\{\lambda a b+b\}$.

(iv) If $z$ is an element of type 4, then $z$ does not cover any element of type 1 or 2.

We now turn our attention to the map $\varphi$.

Lemma 3.5 The map $\varphi: \mathcal{F}\left(\Delta\left([0, n]_{\Lambda}\right)\right) \longrightarrow Q$ is an order-preserving poset map.

Lemma 3.6 For $m<_{Q} \widehat{1}_{Q}$, the collection $\left\{(x, x \cup\{m\}): m \notin x \in \varphi^{-1}(m)\right\}$ is a perfect acyclic matching on the fiber $\varphi^{-1}(m)$.

Thus we have reduced the problem to finding an acyclic matching on the fiber $\Gamma=\varphi^{-1}\left(\widehat{1}_{Q}\right)$.

Lemma 3.7 The collection $\{(x, x \cup\{\lambda a b+b\}): x$ is a chain of type 1 with parameter $\lambda\}$ is an acyclic matching on $\Gamma$ where the critical cells are the chains of type 3 and 4.

Proof: We have seen from parts $(i)$ and $(i i)$ of Lemma 3.4 that to every element $x$ of type 1 there exists a corresponding element $y$ of type 2 with the same parameter and vice-versa. In other words, this is a perfect matching on chains of type 1 and 2. Chains of type 3 and 4 are left unmatched.

We must now show that this matching is acyclic, that is, a directed cycle of the form described in Definition 2.2 cannot exist. Let $z_{1}$ be a chain of type 2 with parameter $\lambda$. Then $d\left(z_{1}\right)=z_{1}-\{\lambda a b+b\}$ is an element of type 1 with the same $\lambda$. Part (iii) of Lemma 3.4 tells us that any $z_{2}$ different from $z_{1}$ will have a smaller parameter. Therefore, we cannot return to $z_{1}$ using our matching. Hence the matching is acyclic.

Lemma 3.8 Let $n=k a b+r$ for $0 \leq r<a b$. If $r=0, a, b$, or $a+b$, then the matching given in Lemma 3.7 has exactly one critical cell. If $r=j b$ for $2 \leq j \leq a-1$, there are exactly two unmatched chains of $\Gamma$. Otherwise, there are no critical cells in $\Gamma$. More precisely, the critical cells of $\Gamma$ are given by

$$
\begin{cases}\left\{D_{k-1}\right\} & \text { if } n=k a b, \\ \left\{C_{k}\right\} & \text { if } n=k a b+a, \\ \left\{C_{k}\right\} & \text { if } n=k a b+b, \\ \left\{D_{k}\right\} & \text { if } n=k a b+a+b, \\ \left\{C_{k}, D_{k}\right\} & \text { if } n=k a b+i b, 2 \leq i \leq a-1, \\ \emptyset & \text { otherwise. }\end{cases}
$$


Proof: The only elements of $\Gamma$ that were not matched are those of type 3 and 4 in Lemma 3.3 . Thus, we need to determine the number of type 3 and 4 elements in $\Gamma$, that is, the number of integers $\lambda$ such that $\lambda a b<_{\Lambda} n$ and $\lambda a b+a \nless_{\Lambda}$ (type 3) and integers $\lambda$ such that $\lambda a b+b<_{\Lambda} n$ and $\lambda a b+a+b k_{\Lambda} n$ (type 4).

Using the Frobenius number, we know that every integer smaller than $n-(a b-a-b)=(k-1) a b+$ $a+b+r$ is comparable with $n$ with respect to the order $<_{\Lambda}$. We do not need to check $\ell a b+a$ or $\ell a b+a+b$ for $0 \leq \ell<k$ because this number is always comparable to $n$ (unless $r=0$ when we must check $(k-1) a b+a+b)$. We also do not need to consider $\ell a b+a$ or $\ell a b+a+b$ for $\ell \geq k+1$ because we would have $\ell a b+a, \ell a b, \ell a b+a+b$, and $\ell a b+b$ all not contained in $[0, n]_{\Lambda}$. Thus, we only need to check $(k-1) a b+a+b$ (if $r=0), k a b+a$, and $k a b+a+b$

There are nine cases to consider.

- $r \notin \Lambda$. Then we have both $k a b+a \nless_{\Lambda} n$ and $k a b \nless_{\Lambda} n$, and also $k a b+a+b \nless_{\Lambda}$ and $k a b+b \nless_{\Lambda} n$. Therefore, there are no critical cells.

Otherwise, $r$ belongs to the semigroup $\Lambda$ and we can write $r=i a+j b$, where $i$ and $j$ are unique nonnegative integers.

- $(i, j)=(0,0)$. We see that $k a b+a \nless_{\Lambda} n$, but also $k a b \nless_{\Lambda} n$. Similarly, we have $k a b+a+b \nless_{\Lambda} n$ and $k a b+b \nless_{\Lambda} n$. Finally, we check and see that $(k-1) a b+a+b \nless_{\Lambda} n$ because $k a b-(k-1) a b+$ $a+b=a b-a-b \notin \Lambda$. Also $(k-1) a b+b<_{\Lambda} n$ because $k a b-(k-1) a b+b=a b-b=(a-1) b \in \Lambda$. Thus we have the one critical cell $D_{k-1}$.

- $(i, j)=(1,0)$. We can easily see that $k a b+a+b \nless_{\Lambda} n$ and $k a b+b \nless_{\Lambda} n$. However, we have $k a b+a \nless_{\Lambda} n$ while $k a b<_{\Lambda} n$. Therefore, we have the one critical cell $C_{k}$.

- $(i, j)=(0,1)$. In this case we again see that $k a b+a+b \nless_{\Lambda} n$ and $k a b+b \nless_{\Lambda} n$. However, we still have $k a b+a \nless_{\Lambda} n$, while $k a b<_{\Lambda} n$. Thus we have the one critical cell $C_{k}$.

- $(i, j)=(1,1)$. First we note that $k a b+a<_{\Lambda} n$. Thus we only check to see that $k a b+a+b k_{\Lambda} n$ and $k a b+b<_{\Lambda} n$. This is easily true, so there is one critical cell $D_{k}$.

$-i=0,2 \leq j \leq a-1$. Clearly $k a b+a \nless_{\Lambda} n$ while $k a b<_{\Lambda} n$. Also, we see that $k a b+a+b \nless_{\Lambda} n$ while $k a b+b<_{\Lambda} n$. Thus the unmatched cells are $C_{k}$ and $D_{k}$.

- $i \geq 1, j \geq 2$. Both $k a b+a$ and $k a b+a+b$ are both comparable with $n$. Therefore there are no critical cells.

- $i \geq 2, j=0$. We see that $k a b+a$ is comparable with $n$. Also, both $k a b+a+b$ and $k a b+b$ are not comparable with $n$. Therefore there are no critical cells.

$-i \geq 2, j=1$. Then $k a b+a$ and $k a b+a+b$ are both comparable with $n$. Therefore there are no critical cells.

Proof of Theorem 3.1: By applying the Patchwork Theorem to the function $\varphi$ we see that the homotopy type of $\Delta\left([0, n]_{\Lambda}\right)$ depends only on the fiber $\varphi^{-1}\left(\widehat{1}_{Q}\right)=\Gamma$. Applying Lemmas 3.7 and 3.8 , there is only one critical cell when $n \equiv 0, a, b, a+b \bmod a b$ and no critical cells in every other case except when 
$i=0$ and $2 \leq j \leq a-1$. However, we claim in this last case we can add the pair $\left(C_{k}, D_{k}\right)$ to the matching on $\Gamma$ and still be left with an acyclic matching.

Lemma 3.4 ( $i v$ ) shows that a chain of type 4 does not cover any chain of type 1 . Hence, when adding the edge $\left(C_{k}, D_{k}\right)$ to the Morse matching of $\Gamma$, it will not create any directed cycles through the chain $D_{k}$. Hence the matching is still acyclic and there are no critical cells in this case.

The critical cells for $n \equiv 0, a, b, a+b \bmod a b$ can be easily seen to be of dimension $2 n / a b-2,2(n-$ $a) / a b-1,2(n-b) / a b-1$, and $2(n-a-b) / a b$, respectively. Therefore, applying the main theorem of reduced discrete Morse theory, Corollary 2.4, proves the result.

\section{Generators in an arithmetic sequence}

Recall that the $q$-analogue is defined as follows: $[a]_{q^{d}}=1+q^{d}+\left(q^{d}\right)^{2}+\cdots+\left(q^{d}\right)^{a-1}$.

Theorem 4.1 Let $\Lambda$ be the sub-semigroup generated by the integers $\{a, a+d, a+2 d, \ldots, a+(a-1) d\}$ where $a$ and $d$ are relatively prime. The order complex of the associated Frobenius interval $[0, n]_{\Lambda}$ is homotopy equivalent to a wedge of spheres where the ith Betti number satisfies

$$
\sum_{n \geq 0} \widetilde{\beta}_{i} q^{n}=q^{a+(i+1)(a+d)} \cdot[a]_{q^{d}} \cdot[a-1]_{q^{d}}^{i+1} .
$$

Example 4.2 For the generators $\{4,5,6,7\}$, that is $a=4$ and $d=1$, we have

$$
\begin{aligned}
& \sum_{n \geq 0} \widetilde{\beta}_{1} q^{n}=q^{14} \cdot[4] \cdot[3]^{2}=q^{14}+\cdots+3 q^{20}+q^{21}, \\
& \sum_{n \geq 0} \widetilde{\beta}_{2} q^{n}=q^{19} \cdot[4] \cdot[3]^{3}=q^{19}+4 q^{20}+\cdots+q^{28},
\end{aligned}
$$

and no other generating polynomial contains the $q^{20}$ term. Hence the Frobenius complex $\Delta\left([0,20]_{\Lambda}\right)$ is homotopy equivalent to a wedge of three circles and four 2 -spheres.

The Frobenius number of an arithmetic sequence was given in equation (1). Therefore, for the generators $\{a, a+d, a+2 d, \ldots, a+(a-1) d\}$, we have the Frobenius number

$$
\left(\left\lfloor\frac{a-2}{a-1}\right\rfloor+1\right) \cdot a+(d-1)(a-1)-1=(a-1) d .
$$

We will proceed as before and use Discrete Morse theory and the Patchwork theorem. Let $A$ be the set $\{a+d, a+2 d, \ldots, a+(a-1) d\}$.

Definition 4.3 Given $n$, let $R$ be the chain $\{1,2,3,4, \ldots, n-a\}$ with a maximal element $\widehat{1}_{R}$ adjoined. That is,

$$
R=\{1,2,3, \ldots, n-a\} \cup\left\{\widehat{1}_{R}\right\}
$$


If $x=\left\{x_{1}, x_{2}, \ldots, x_{k}\right\} \in \Delta\left([0, n]_{\Lambda}\right)$ and we define $x_{0}=0$, let $\psi: \mathcal{F}\left(\Delta\left([0, n]_{\Lambda}\right)\right) \rightarrow R$ be a map defined by

$$
\psi(x)=\left\{\begin{array}{cl}
x_{i-1}+a, \quad x_{i}-x_{i-1} \notin A, \\
\\
x_{j}-x_{j-1} \in A \\
& \text { for } 1 \leq j \leq i-1, \\
x_{k}+a, & n-x_{k} \notin\{a\} \cup A, \\
& x_{j}-x_{j-1} \in A \\
& \text { for } 1 \leq j \leq k, \\
\widehat{1}_{R}, \quad & \text { otherwise. }
\end{array}\right.
$$

Lemma 4.4 The element $m \cdot d$ is not contained in $\Lambda$ for $1 \leq m \leq(a-1)$.

Proof: Suppose $m \cdot d \in \Lambda$ for $1 \leq m \leq(a-1)$. Then for $0 \leq s_{i} \leq(a-1)$, we have

$$
\begin{aligned}
m \cdot d & =\left(a+s_{1} d\right)+\left(a+s_{2} d\right)+\cdots+\left(a+s_{k} d\right) \\
& =k a+\left(s_{1}+\cdots+s_{k}\right) d .
\end{aligned}
$$

The fact that $d$ and $a$ are relatively prime implies that $d$ divides $k$. That is, $k=\ell \cdot d$. Therefore, $m=\ell \cdot a+\left(s_{1}+\cdots+s_{k}\right)$ which implies that $m \geq a$. This is a contradiction.

Lemma 4.5 Let $x_{i}$ and $x_{j}$ be elements of a chain $x$ such that $x_{i}-x_{j} \in\{a\} \cup A$. Then the open interval $\left(x_{i}, x_{j}\right)_{\Lambda}$ is empty.

The following lemma is an immediate consequence of Lemma 4.5 and the definition of the function $\psi$.

Lemma 4.6 If $x$ and $y$ are chains such that $x \subseteq y$ and $\psi(x)=x_{i-1}+a$ then $x_{j}=y_{j}$ for $1 \leq j \leq i-1$. In particular, if $\psi(x)=\widehat{1}_{R}$ then $x=y$.

We can finally give a few properties of the map $\psi$.

Lemma 4.7 The map $\psi: \mathcal{F}\left(\Delta\left([0, n]_{\Lambda}\right)\right) \rightarrow R$ is an order preserving poset map.

Lemma 4.8 For $m<_{R} \widehat{1}_{R}$, the collection $\left\{(x, x \cup\{m\}): m \notin x \in \psi^{-1}(m)\right\}$ is a perfect acyclic matching on the fiber $\psi^{-1}(m)$.

Proof: Suppose $\psi(x)=x_{i-1}+a$ and $x_{i-1}+a \in x$. That is, $x_{i}=x_{i-1}+a$. It is clear that $d(x)=x-\left\{x_{i}\right\}$ is a valid chain in the Frobenius complex since we are simply removing an element. We need to check that $\psi(d(x))=x_{i-1}+a$. We know that $d(x)_{j}-d(x)_{j-1}=x_{j}-x_{j-1} \in A$ for $1 \leq j \leq(i-1)$. Suppose $d(x)_{i}-d(x)_{i-1}=x_{i+1}-x_{i-1} \in A$. Then, by Lemma 4.5. $\left(x_{i-1}, x_{i+1}\right)_{\Lambda}$ would have to be empty. This contradicts the fact that $x_{i} \in\left(x_{i-1}, x_{i+1}\right)_{\Lambda}$ in the chain $x$. Since $d(x)_{i}-d(x)_{i-1} \notin A$ and $d(x)_{j}-d(x)_{j-1} \in A$ for $1 \leq j \leq(i-1)$, we have $\psi(d(x))=d(x)_{i-1}+a=x_{i-1}+a$.

Now suppose that $\psi(x)=x_{i-1}+a$ and $x_{i-1}+a \notin x$. It is clear that $u(x)=x \cup\left\{x_{i-1}+a\right\}$ would be mapped to $x_{i-1}+a$. Thus, it must be shown that $u(x)$ is a valid chain, that is, $x_{i-1}+a$ is comparable to $x_{i}$. We know that $x_{i}-x_{i-1} \notin A$. Suppose

$$
x_{i}-x_{i-1}=\left(a+s_{1} d\right)+\left(a+s_{2} d\right)+\cdots+\left(a+s_{k} d\right)
$$


where $s_{1} \leq s_{2} \leq \cdots \leq s_{k}$ and $k \geq 2$. Then

$$
x_{i}-\left(x_{i-1}+a\right)=\left(a+\left(s_{1}+s_{2}\right) d\right)+\left(a+s_{3} d\right)+\cdots+\left(a+s_{k} d\right) .
$$

If $s_{1}+s_{2} \leq a-1$, then we have written this difference as a sum of generators. Therefore, $x_{i}$ and $x_{i-1}+a$ are comparable.

If $s_{1}+s_{2}>a-1$, then the difference is larger than $(a-1) d$, which is the Frobenius number of the generators. Thus, $x_{i}$ and $x_{i-1}+a$ are comparable. Therefore, $u(x)$ is a valid chain.

Finally, the matching on the fiber is clearly acyclic since the same element is either added or removed from a chain.

Using the Patchwork theorem, we have an acyclic matching on $\mathcal{F}\left(\Delta\left([0, n]_{\Lambda}\right)\right)$ whose only critical cells are the elements of the fiber $\psi^{-1}\left(\widehat{1}_{R}\right)$. Note that Lemma 4.6 says that each of these cells are maximal. Therefore, due to Theorem 2.5 , we will have that $\Delta\left([0, n]_{\Lambda}\right.$ is homotopy equivalent to a wedge of spheres whose number and dimension corresponds to the number and dimension of the critical cells. Thus, we are interested in counting the number of chains that are mapped to $\widehat{1}_{R}$. The following lemma is straightforward from the definition of the function $\psi$.

Lemma 4.9 The fiber $\psi^{-1}\left(\widehat{1}_{R}\right)$ consists of elements $x=\left\{x_{1}, x_{2}, \ldots, x_{k}\right\}$ where $x_{i}-x_{i-1} \in A$ for $1 \leq i \leq k$ and $n-x_{k} \in\{a\} \cup A$.

Proof of Theorem 4.1: We know from Lemma 4.9 that the critical cells are in bijection with compositions of $n$ where the last part belongs to the set $\{a\} \cup A$ and the remaining parts belong to the set $A$. Furthermore if such a composition has $i+2$ parts, it will contribute to the $i$-dimensional homology. Hence, fixing $i$, we obtain the generating function

$$
\begin{aligned}
\sum_{n \geq 0} \widetilde{\beta}_{i} q^{n} & =\left(\sum_{k=0}^{a-1} q^{a+k d}\right) \cdot\left(\sum_{\ell=1}^{a-1} q^{a+\ell d}\right)^{i+1} \\
& =q^{a+(i+1)(a+d)} \cdot\left(\sum_{k=0}^{a-1} q^{k d}\right) \cdot\left(\sum_{\ell=0}^{a-2} q^{\ell d}\right)^{i+1} \\
& =q^{a+(i+1)(a+d)} \cdot[a]_{q^{d}} \cdot[a-1]_{q^{d}}^{i+1}
\end{aligned}
$$

\section{Concluding remarks}

The Frobenius poset generated by two relatively prime integers can be embedded on a cylinder. There are many results (see, for example, [3, 6]) on posets that can be embedded in the plane. Can any of these results be extended to cylindrical posets?

There are other classes of generators, such as a geometric sequence, that have closed formulas for the Frobenius number, see [15]. Does the Frobenius complex have a nice topological representation in this case?

More generally, all computational evidence suggests that the Frobenius complex - even for randomly selected generators - has a relatively simple topology, that is, it is torsion-free. Is there a set of generators that creates torsion in the associated Frobenius complex? 


\section{Acknowledgements}

The authors would like to thank Richard Stanley for pointing out the references [5, 10, 16] and Volkmar Welker for suggesting Corollary 3.2. We also thank Benjamin Braun and Margaret Readdy who read earlier versions of this paper.

\section{References}

[1] A. BARVInok, “A Course in Convexity,” American Mathematical Society, 2002.

[2] M. Beck And S. Robins, “Computing the Continuous Discretely,” Springer, 2007.

[3] L. J. Billera And G. Hetyei, Decompositions of partially ordered sets, Order 17 (2000), 141166.

[4] L. J. Billera AND A. N. Myers, Shellability of interval orders, Order 15 (1999), 113-117.

[5] A. BJÖRNER AND V. WELKER, Segre and Rees products of posets, with ring-theoretic applications, J. Pure Appl. Algebra 198 (2005), 43-55.

[6] K. L. Collins, Planar lattices are lexicographically shellable, Order 8 (1992), 275-381.

[7] R. Ehrenborg And G. Hetyei, The topology of the independence complex, European J. Combin. 27 (2006), 906-923.

[8] R. Forman, Morse theory for cell complexes, Adv. Math. 134 (1998), 90-145.

[9] R. Forman, A user's guide to Morse theory, Séminaire Lotharingien de Combinatoire 48 (2002), Article B48c.

[10] P. HeRsh AND V. Welker, Gröbner basis degree bounds on $\operatorname{Tor}^{k[\Lambda]}(k, k)$ and discrete Morse theory for posets, in Integer points in polyhedra-geometry, number theory, algebra, optimization, pp. 101-138, Contemp. Math., 374, Amer. Math. Soc., Providence, RI, 2005.

[11] D. N. Kozlov, Complexes of directed trees, J. Combin. Theory Ser. A 88 (1999), 112-122.

[12] D. N. KozLov, “Combinatorial Algebraic Topology,” Springer, 2008.

[13] D. N. Kozlov, Discrete Morse theory and Hopf bundles, preprint 2009.

[14] O. A. Laudal And A. Sletsjøe, Betti numbers of monoid algebras. Applications to 2dimensional torus embeddings, Math. Scand. 56 (1985), 145-162.

[15] D. Ong And V. Ponomarenko, The Frobenius number of geometric sequences, Integers 8 (2008), A33.

[16] I. Peeva, V. Reiner, B. Sturmfels, How to shell a monoid, Math. Ann. 310 (1998), 379-393.

[17] J. B. Roberts, Note on linear forms, Proc. Amer. Math Soc. 7 (1956), 456-469. 